\title{
Human Anterior Intraparietal and Ventral Premotor Cortices Support Representations of Grasping with the Hand or a Novel Tool
}

\author{
Stéphane Jacobs*, Claudia Danielmeier ${ }^{* *}$, and Scott H. Frey
}

\begin{abstract}
Humans display a remarkable capacity to use tools instead of their biological effectors. Yet, little is known about the mechanisms that support these behaviors. Here, participants learned to grasp objects, appearing in a variety of orientations, with a novel, handheld mechanical tool. Following training, psychophysical functions relating grip preferences (i.e., pronated vs. supinated) to stimulus orientations indicate a reliance on distinct, effectorspecific internal representations when planning grasping actions on the basis of the tool versus the hands. Accompanying fMRI data show that grip planning in both hand and tool conditions was associated with similar increases in activity within the same regions of the anterior intraparietal and caudal ventral premotor cortices, a putative homologue of the macaque anterior intraparietalventral premotor (area F5) "grasp circuit." These findings suggest that tool use is supported by effector-specific representations of
\end{abstract}

\section{INTRODUCTION}

An extraordinary property of the nervous system is the ability to perform the same action using different effectors. For instance, objects can be grasped with the hands, mouth, or even the toes. Tool use exemplifies an extension of this capacity, known as "motor equivalence" (Wing, 2000; Keele, Cohen, \& Ivry, 1990; Lashley, 1930), to nonbiological effectors. With training, humans can become proficient at grasping objects with a variety of handheld tools including tweezers, pliers, tongs, and even remotely operated devices such as cranes and robotic arms. Do these technological behaviors arise from experiencedependent changes within the same neural mechanisms that underlie manual grasping? Or do they recruit other neural systems?

The cerebral bases of manual prehension have been extensively explored in previous investigations (Castiello \& Begliomini, 2008). There is considerable evidence that grasping actions in monkeys are coded within a circuit involving the anterior intraparietal (AIP) area belonging to

University of Oregon

*Current address: Space and Action, INSERM U864, Bron, France.

**Current address: Max Planck Institute for Neurological Research, Cologne, Germany. grasping with the tool that are functionally independent of previously existing representations of the hand and yet occur within the same parieto-frontal regions involved in manual prehension. These levels of representation are critical for accurate planning and execution of actions in a manner that is sensitive to the respective properties of these effectors. These effector-specific representations likely coexist with effector-independent representations. The latter were recently reported in macaque F5 [Umiltà, M. A., Escola, L., Intskirveli, I., Grammont, F., Rochat, M., Caruana, F., et al. When pliers become fingers in the monkey motor system. Proceedings of the National Academy of Sciences, U.S.A., 105, 2209-2213, 2008] and appear to be established by tool use training through modification of existing representations of grasping with the hand. These more abstract levels of representation may facilitate the transfer of skills between hand and tool.

the inferior parietal lobule and interconnected area $\mathrm{F} 5$ located in the ventral premotor (PMv) cortex (Jeannerod, Arbib, Rizzolatti, \& Sakata, 1995). This pathway is implicated in transforming objects' intrinsic spatial properties into motor programs for grasping (Fogassi et al., 2001). Functional neuroimaging (Frey, Vinton, Norlund, \& Grafton, 2005; Culham et al., 2003; Ehrsson, Fagergren, \& Forssberg, 2001; Binkofski et al., 1999) and transcranial magnetic stimulation (Davare, Andres, Clerget, Thonnard, \& Olivier, 2007; Davare, Andres, Cosnard, Thonnard, \& Olivier, 2006; Rice, Tunik, \& Grafton, 2006; Tunik, Frey, \& Grafton, 2005) studies in humans suggest that the anterior inferior parietal lobule, within and along the anterior intraparietal sulcus (aIPS), and ventral precentral gyrus (putative PMv) may constitute a homologue of the monkey parieto-frontal grasp circuit.

Motor equivalence is believed to be supported by abstract, effector-independent representations of action. This interpretation is based on the presence of kinematic regularities when the same action (e.g., writing) is undertaken with different biological effectors (i.e., the hand or the toe) (Wing, 2000; Keele et al., 1990). By extension, previous neuroimaging studies have interpreted areas that show increased activation when the same action is performed with different biological effectors as supporting effector-independent representations (Castiello et al., 
2000; Rijntjes et al., 1999). Kinematic regularities have also been observed during grasping actions performed with a tool or the hand (Gentilucci, Roy, \& Stefanini, 2004), raising the possibility that effector-independent representations may also underlie the emergence of motor equivalence between biological and nonbiological effectors. Consistent with this view, recent findings in macaques suggest that single cells in area F5, that initially code manual prehension, come to also represent grasping actions performed with a tool after extensive training (Umiltà et al., 2008).

Although these findings suggest that newly learned tool use actions are supported by effector-independent representations within areas involved in the control of manual actions, the role played by the parieto-frontal grasp circuit in representing these actions remains unknown. Likewise, it is uncertain whether similar mechanisms underlie the acquisition of novel tool use behaviors in human beings who, unlike macaques, are natural users of technology. To address these issues, we first trained participants to grasp objects using a novel handheld tool whose end-effector mechanics differed from those of the hand (Figure 1). Then, variations in the BOLD signal, which provides a measure of local neural activity (Logothetis, Pauls, Augath, Trinath, \& Oeltermann, 2001), were recorded using fMRI while participants planned grasping actions on the basis of the use of either their hands or the tool in the absence of overt execution.

\section{METHODS}

\section{Participants}

Twenty healthy participants (12 women, age range $=19$ 41 years, mean $=24.07$ years) gave their informed consent to participate in this study. All were right-handed, as assessed by the Edinburgh Handedness Inventory (Oldfield, 1971), and had normal or corrected-to-normal visual acuity.

\section{Experimental Design}

Participants took part in two experimental sessions, each following a 2 (Effector Type: hand, tool) $\times 2$ (Effector Side: left, right) factorial design, resulting in four conditions: Hand Left, Tool Left, Hand Right, and Tool Right. In Session 1, participants practiced an overt grip selection (OGS) task in which they were required to reach and grasp a stimulus object presented in various orientations in the most comfortable manner, using either an overhand or an underhand grip, that is, a pronated or a supinated posture of the hand (Figure 2A). This task was performed with the hands, using a precision grip, and with a handheld mechanical tool. Importantly, when using this tool to grasp the stimulus object, the movements and the postures of the hands differed from manual grasping in two ways (Figure 1A and B). First, the tool was held with a power grasp, and the whole hand flexion or extension caused the jaws of the tool to close or open, respectively. Second, the opposition axis between the jaws of the tool was offset by $90^{\circ}$ relative to that of the hand (Figure 1B). In Session 2, participants performed a prospective grip selection (PGS) task while neural activity was recorded from the entire brain using BOLD fMRI. This PGS task was identical to the OGS task except that participants remained still while deciding whether an over- or underhand grip would be preferred when grasping stimulus objects using the hands or tool (Figure 1). Previous work indicates that PGS involves movement planning to choose the least awkward grip (Johnson et al., 2002; Johnson, 2000).

\section{OGS Task: Behavioral Pretesting (Session 1)}

Participants practiced grasping stimulus objects with the hands and with the tool, and their grip preferences (i.e., over- or underhand grips) were recorded. The mechanical tool was $55.9 \mathrm{~cm}$ long and weighed $170 \mathrm{~g}$. Participants were seated at a table in front of a computer screen that was easily reached either with the free hand or with the tool, depending on the condition (see below). For consistency, the same 2-D stimulus objects were used in both the OGS and the PGS tasks. Stimuli consisted of a graphically rendered sphere with photo-realistic shading to provide the illusion of three-dimensionality (Figure 1A). The sphere was half pink and half tan, with indentations on each side for "finger placement." Stimuli appeared against a black background in 24 orientations ( $15^{\circ}$ increments) rotated around the line-of-sight $(z)$ axis. To create 3 -D objects for grasping, we back projected the stimuli through a transparent plastic overlay fitted over the surface of a flat computer monitor (Figure 1B). The center of the overlay was a 3-D transparent plastic disk that extended $25 \mathrm{~mm}$ from the surface of the computer screen. This disk was equivalent in size (32 $\mathrm{mm}$ radius) to the projected stimulus object, creating the appearance of a graspable object extending from the surface of the screen.

Participants completed two runs of 192 trials each. The tool was held by the same hand throughout the first run and then switched to the other hand for the second run. The hand (side) holding the tool in the first run was counterbalanced across participants. Each run was divided into eight blocks, with the side tested (one side with a free hand, the other holding the tool) alternating across blocks, so that participants had to use either their free hand or the tool during an entire block. The first side tested in each block was counterbalanced across participants.

A block started by a leftward- or a rightward-pointing arrow presented centrally to indicate the side to use for the forthcoming trials (i.e., the free hand or the one holding the tool). Then, 24 trials were presented (one per stimulus orientation; $0^{\circ}-345^{\circ}$ in $15^{\circ}$ increments) in random order for $4000 \mathrm{msec}$ each. On each trial, participants were instructed to start with their cued hand in a neutral position 
Figure 1. Experiment setup and design. (A) Novel tool and a sample of stimulus object orientations. (B) The overt grip selection (OGS) task.

Participants first practiced grasping the stimulus object in a variety of orientations. Depending on the condition, grasping was performed with the left or right hand, using a precision grip, or with the tool operated with either hand. The Tool Right condition is illustrated. (C) Structure of a single trial of the prospective grip selection (PGS) task. After completing the OGS task, participants were asked to select grips based on the use of their hands and the tool in the absence of overt movements. Specifically, they had to decide on which colored indentation their thumb or the target jaw (i.e., upper or lower, balanced across participants) of the tool would be on the object if they were to grasp it using the most comfortable posture (i.e., undervs. overhand). Associated brain activity was recorded using BOLD fMRI. A response cue (pink and tan squares, position varied randomly) appeared after the stimulus object to indicate which foot pedal to press to give the desired answer. This trial-by-trial variation of the response mapping delayed selection and execution of foot press responses and thus enabled us to separate the associated neural activity

from that which accompanied the earlier grip selection. The red rectangle represents the period modeled in the analysis of fMRI data (onset of the stimulus object through first $3000 \mathrm{msec}$ of delay interval), which corresponds only to the planning of grasping actions. A small circle at the bottom of the screen reminded the participant what limb (left or right) to base their decision on. Variable durations of the different phases within one trial are given (for details, see the Methods section). OBV = onset of brain volume acquisition. (D) Example sequence of events in the PGS task. Within each fMRI run, the tool was held in one hand, and the side used (i.e., empty hand or tool) was alternated between blocks of 10 trials. Each block started with an arrow cuing which side to use for the forthcoming trials, and a dot located at the bottom left or right of the screen stayed throughout the block as reminder (for further details, see the Methods section).

(Figure 2A) with its lateral side placed down on the table and to reach for and grasp the object (with either the free hand or the tool) using the most comfortable posture (i.e., over- or underhand). They were told to use a precision grip, placing their thumb and forefinger, or the jaws of the tool, on the object's indentations. Although the plastic overlay did not have actual indentations, the experimenter reminded the participant when necessary to place his or her fingers, or the jaws of the tool, precisely on the projected indentations (no trials were discarded on the basis of this criterion). The hand was then returned to the starting posture in preparation for the next trial. At the end of each trial, the experimenter coded the side of the object (i.e., pink or tan) on which the thumb or the upper jaw of the tool was placed. This code therefore indicated whether an over- or underhand grip was chosen. At the end of Session 1, participants also completed two practice runs of the PGS task, as detailed below.

\section{PGS Task: fMRI Experiment (Session 2)}

All volunteers completed this testing session within $48 \mathrm{hr}$ after Session 1. Participants reclined on the scanner bed in a supine position with their arms extended along the 
sides of the body. As in Session 1, their hands were oriented in a neutral position (with their lateral sides resting on the scanner bed; Figure 2A), and one was passively holding the tool. The tool was resting on the scanner bed and taped to the participant's leg so that no force was needed to hold it stable. Stimulus objects were back projected onto a screen and viewed through a mirror attached to the head coil. Each participant performed two practice runs of the PGS task in a mock MRI scanner immediately before the actual fMRI session.

Participants held the tool in the same hand during an entire run and switched it to the other hand between runs. The hand holding the tool in the first run was counterbalanced across participants. Therefore, each run was comprised of only two conditions, either Hand Left and Tool Right or Tool Left and Hand Right. Fifteen participants completed three runs of each type, whereas two of each type were completed by the other five individuals. Within each run, the side tested (empty hand or tool) alternated across blocks.

In contrast to the OGS task used in Session 1, participants did not actually grasp the objects during PGS but were instead instructed to remain still during the whole procedure. During hand blocks, they were asked to decide whether they would place their thumb on the pink or tan indentation of the stimulus object (i.e., over- or underhand grasp) if they were to grasp it in the most natural (i.e., comfortable) posture using a precision grip. Similarly, during tool blocks, participants determined whether it would be more natural to place the target jaw of the tool (upper jaw for half of subjects, lower jaw for the other half) on the pink or tan indentation. To avoid potential conflict, participants issued responses by pressing either the "pink" or the "tan" foot pedal (see below) and we converted those to over- or underhand grasps off-line.

Each run consisted of six blocks of 10 trials and the side tested (free hand or tool) alternated across blocks. In addition to the side cue (arrow) presented at the beginning of the block (2000 msec), a small circle at the bottom left or right of the screen remained visible throughout the entire block as a reminder of the cued limb (Figure 1C). A central fixation cross remained visible throughout the entirety of the run, and participants were instructed to maintain fixation.

Each block consisted of 30 randomly ordered trials of each of the two conditions in that run (i.e., Hand Right and Tool Left or Hand Left and Tool Right). Of these 30 trials, 24 were 8000 msec experimental trials (only 12 orientations of the object were used during fMRI- $15^{\circ}$ to $345^{\circ}$ in $30^{\circ}$ increments-each repeated twice), and 6 were 8000 msec null events with only the fixation cross on the black background.

As illustrated in Figure 1C, PGS trials consisted of four phases optimized for rapid, event-related fMRI testing: (1) to increase temporal resolution, an oversampling method (Miezin, Maccotta, Ollinger, Petersen, \& Buckner, 2000) was used in which stimulus onset occurred at a vari- able delay $(0,500,1000$, or $1500 \mathrm{msec})$ relative to the onset of the acquisition of a volume of functional MRI data; (2) a stimulus object was presented in one of the 12 different orientations for a variable duration (i.e., jitter: 3000, 3500, or $4000 \mathrm{msec}$ ); (3) a 2000-msec duration response cue indicating the identity of the mapping between the response pedals (left or right) and the colors (pink or tan) denoting the would-be location of the thumb or tool's target jaw during the selected grip. To avoid the development of heuristics, we counterbalanced the stimulus orientation and the identities of the response pedals across trials for each run. Varying the identity of this response mapping randomly across trials forced the participants to wait for this cue to select and execute their foot response, allowing us to separate neural activity related to grip selection from that associated with foot press selection and execution; and (4) a variable length (500-3000 msec) intertrial interval was used to make the duration of all trials equal $8000 \mathrm{msec}$. Oversampling and jitter durations were counterbalanced within each run.

\section{MRI Procedure}

All scans were performed on a Siemens (Erlangen, Germany) 3T Allegra MRI scanner at the Robert and Beverly Lewis Center for Neuroimaging at the University of Oregon. BOLD echo-planar images were then collected using a T2*-weighted gradient-echo sequence, a standard birdcage radiofrequency coil, and the following parameters: repetition time $=2000 \mathrm{msec}$, echo time $=30 \mathrm{msec}$, flip angle $=$ $80^{\circ}, 64 \times 64$ voxel matrix, field of view $=200 \mathrm{~mm}, 32$ contiguous axial slices acquired in interleaved order, thickness $=$ $3.5 \mathrm{~mm}$, in-plane resolution $=3.125 \times 3.125 \mathrm{~mm}$, and bandwidth $=2605 \mathrm{~Hz} /$ pixel. The initial four scans in each run were discarded to allow steady-state magnetization to be approached. High-resolution T1-weighted structural images were also acquired using the 3-D MP-RAGE pulse sequence: repetition time $=2500 \mathrm{msec}$, echo time $=4.38 \mathrm{msec}$, inversion time $=1100 \mathrm{msec}$, flip angle $=8.0^{\circ}, 256 \times 256$ voxel matrix, field of view $=256 \mathrm{~mm}, 176$ contiguous axial slices, thickness $=1.0 \mathrm{~mm}$, and in-plane resolution $=1 \times 1 \mathrm{~mm}$. DICOM image files were converted to NIFTI format using MRIConvert software (http://lcni.uoregon.edu/ jolinda/ MRIConvert/).

\section{fMRI Data Analyses}

fMRI data were analyzed using tools from the fMRIB Software Library (FSL v.3.3; http://www.fmrib.ox.ac.uk/fsl/). Image preprocessing involved several steps. Motion was corrected using the tools of the FMRIB Software library (Jenkinson, Bannister, Brady, \& Smith, 2002), and nonbrain structures were removed using BET (Smith, 2002). The data were spatially smoothed using a Gaussian kernel of 5-mm FWHM. For each data set, intensity normalization using "grand mean scaling" was applied, whereby each volume in the data set is scaled by the same factor to allow for 


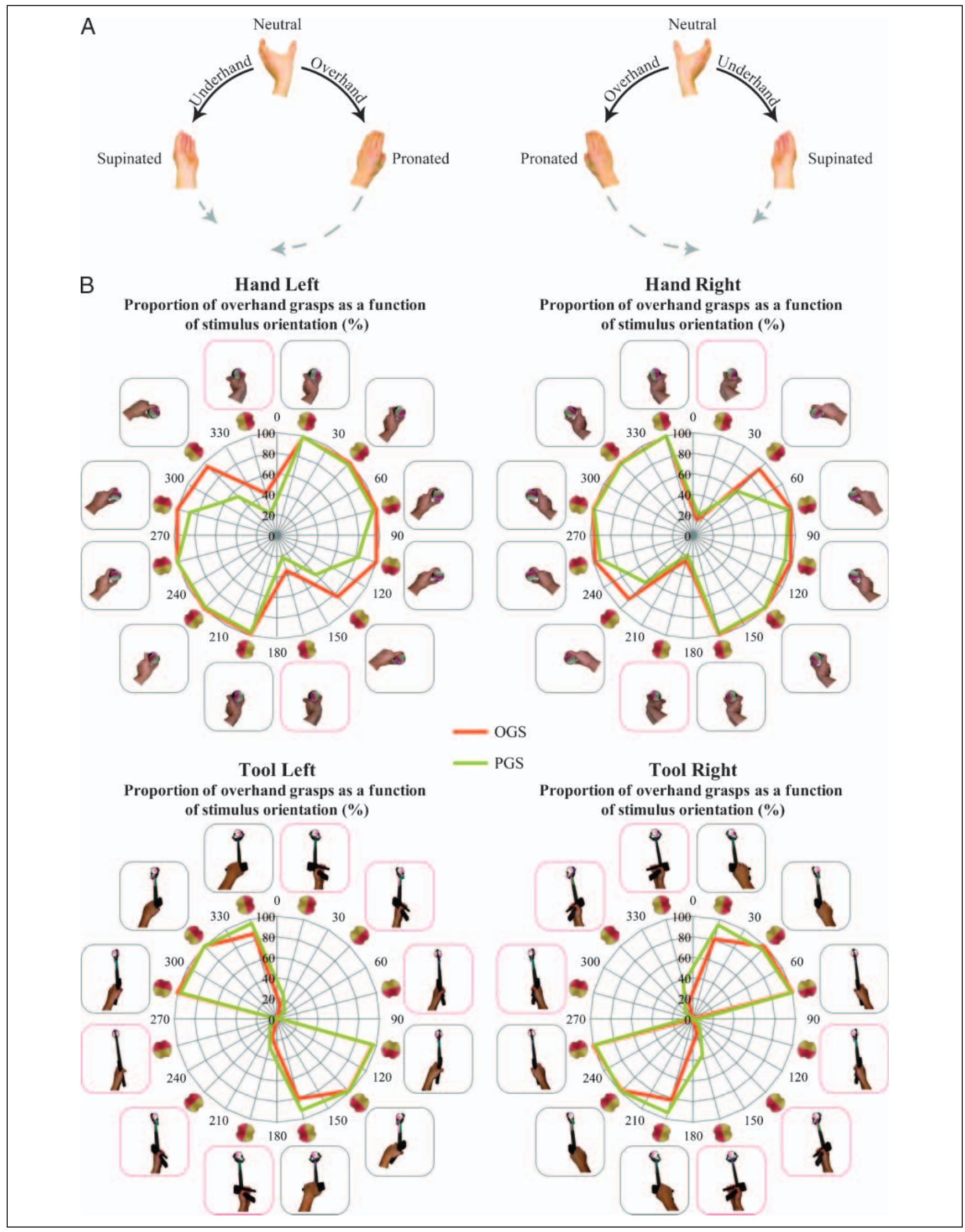


cross-sessions and cross-subjects statistics to be valid. Highpass temporal filtering with a 50-sec cutoff was used to remove low-frequency artifacts. Time series statistical analysis was carried out in FEAT v.5.63 using FILM with local autocorrelation correction (Woolrich, Ripley, Brady, \& Smith, 2001). Delays and undershoots in the hemodynamic response were accounted for by convolving the model with a double-gamma basis function. Registration to high-resolution and standard images (Montreal Neurological Institute template) was implemented using FLIRT (Jenkinson et al., 2002; Jenkinson \& Smith, 2001).

For each participant, fMRI runs containing either Hand Left and Tool Right or Hand Right and Tool Left conditions were modeled separately at the first level. Three explanatory (predictor) variables (EVs) were modeled along with their temporal derivatives. Two EVs coded the experimental conditions, left-side grip selection (i.e., Hand Left or Tool Left), and right grip selection (i.e., Tool Right or Hand Right). These were locked to the onset of the stimulus objects and included the subsequent $3000 \mathrm{msec}$ of the delay period. A third EV coded the 8000-msec null trials that were used as resting baseline. Orthogonal contrasts (one-tailed $t$ tests) were used to test for differences between each of the four experimental conditions and resting baseline.

The resulting first-level contrasts of parameter estimates (COPEs) then served as input to higher level analyses carried out using FLAME Stage 1 (Woolrich, Behrens, Beckmann, Jenkinson, \& Smith, 2004; Beckmann, Jenkinson, $\&$ Smith, 2003) to model and to estimate random-effects components of mixed-effects variance. $Z$ (Gaussianized $T$ ) statistic images were thresholded using clusters determined by $Z>3.1$ and a corrected cluster significance threshold of $p=.05$ (Worsley, Evans, Marrett, \& Neelin, 1992), unless indicated otherwise. First, a whole-brain analysis was undertaken to identify the cerebral areas that responded significantly to the experimental conditions when compared with resting baseline at the group level. The first-level COPEs were averaged across runs for each subject separately (second level) and then across participants (third level) (see the Planning grasping actions with the hands or tool compared with resting baseline section). Second, to test for the main effects of Effector Side and Effector Type and for the interaction between these two factors, a standard 2 (Effector Side: left, right) $\times 2$ (Effector Type: hand, tool) repeated measures ANOVA ( $F$ tests) was carried out on first-level COPEs (see the Main effects of Effector Type and Effector Side section). To increase the sensitivity of this analysis by reducing the number of multiple statistical comparisons, it was restricted to only those voxels that showed a significant increase in activity in at least one of the four experimental conditions compared with resting baseline at the group level of the whole-brain analysis $(Z>3.1$, corrected cluster significance threshold of $p=.05$ ).

Verification of anatomical localization of brain activations was undertaken by manual comparison with an atlas (Duvernoy, 1991). In addition, Freesurfer software (http://surfer.nmr.mgh.harvard.edu/) was used to reconstruct individual brain surfaces from individual's highresolution structural scans (Segonne et al., 2004; Dale, Fischl, \& Sereno, 1999; Fischl, Sereno, \& Dale, 1999) and to create an average brain surface representative of the anatomy of our sample of subjects (Fischl, Sereno, Tootell, \& Dale, 1999). Group statistical maps were then overlayed on this average brain surface.

\section{ROI Analysis}

To characterize the nature of the changes in activation induced within the grasp circuit by the PGS task better, we centered spherical ROIs on the peak activations revealed in bilateral aIPS and left PMv in the whole-brain analysis comparing each of the four conditions with resting baseline. These activation peaks were located at virtually identical coordinates in all four conditions (Table 1). Therefore, ROIs were centered on the mean $x, y, z$ coordinates obtained by averaging across conditional peaks (Table 1). Mean percent signal change relative to the resting baseline was calculated for each participant and each condition within these ROIs by taking the ratio of the COPE (experimental condition $>$ rest) and the mean voxel intensity and scaling by the peak height of a regressor formed by convolving an isolated 3-sec event with the double gamma hemodynamic response function. This ensures accurate

\footnotetext{
Figure 2. Grip preferences during overt grip selection (OGS) and prospective grip selection (PGS). (A) Representation of the neutral, pronated, and supinated postures of the left and right hand and their relation to the definition of over- and underhand grasps. The limits of range of motion in each direction are represented by the dotted arrows. (B) Polar plots of the average proportion of overhand grasps across subjects for each orientation of the stimulus object, as observed in the OGS (orange) and PGS (green) tasks. A data point to the periphery of the plot represents 100\% of overhand grips, whereas a data point at the center of the plot means that $100 \%$ of the grips selected for that orientation were underhand. The stimulus object is shown in each of the tested orientations $\left(15^{\circ}-345^{\circ}\right.$ in $30^{\circ}$ increments), and the corresponding preferred grasp (i.e., grip selected on $>50 \%$ of trials) is represented next to it. A gray frame around the picture representing the preferred grip indicates an overhand grasp, whereas underhand grips are signaled by a red frame. In both Hand Left and Hand Right conditions, participants used mostly overhand grasps, except at the limits of their range of motion in the pronation direction. The functions describing the variation of grip preferences across stimulus orientation are $180^{\circ}$ out of phase between Hand Left and Hand Right, reflecting the fact that joint constraints of the two arms are mirror images. The same is true for Tool Left versus Tool Right conditions. Consistent with reliance on effector-specific internal models, grip preferences for use of the hands versus tools differ substantially on both the left and the right sides. In both tool use conditions, participants preferred underhand grasps for fully half of the stimulus orientations. Critically, in all four conditions, the functions describing grip preferences observed in OGS and PGS tasks were virtually identical, indicating that participants prospectively selected grips in the PGS task that were consistent with their overt behavior in the OGS task.
} 
Table 1. Coordinates of the Peak Activations Revealed in Bilateral aIPS and Left PMv by the Whole-Brain Analysis for Each of the Four Conditions in the Design

\begin{tabular}{lccr}
\hline & & ROI & \\
\cline { 2 - 4 } Condition & Left aIPS $(x, y, z)$ & Right aIPS $(x, y, z)$ & -56236 \\
\hline Hand Left & $-34-4444$ & $40-3444$ & Left PMv $(x, y, z)$ \\
Tool Left & $-40-4446$ & NA & NA \\
Hand Right & $-40-4446$ & NA & -54238 \\
Tool Right & $-36-4444$ & $40-3444$ & -56236 \\
Center of the ROI (average) & $-38-4445$ & & -56236 \\
\hline
\end{tabular}

The center of each spherical ROI was obtained by averaging these coordinates across conditions. Coordinates $(x, y, z)$ expressed in millimeters in the standard space of the Montreal Neurological Institute template (MNI-152).

$\mathrm{NA}=$ no significant activation detected.

interpretation of the percent change of COPEs outside of the particular context of a given study (Mumford, 2007). Mean time course of the signal within these ROIs was also computed in terms of percent change from resting baseline for each subject and condition (see Figure S1 provided as Supplementary material on-line). This was done by calculating the ratio of voxel intensity and the mean voxel intensity for each voxel in the ROI and for each data point within a 14-sec window around stimulus onset (every $500 \mathrm{msec}$ from $2 \mathrm{sec}$ before stimulus onset through $12 \mathrm{sec}$ after stimulus onset). The 500-msec resolution was possible because of the use of the oversampling design described above.

\section{RESULTS}

\section{Behavioral Results}

The proportion of overhand grasps for every stimulus orientation was calculated for each participant and condition separately for both the OGS and the PGS tasks and submitted to repeated measures ANOVA. Consistent with previous work (Johnson, 2000), the functions describing grip preferences across stimulus orientations for the two hands in the OGS task were $180^{\circ}$ out of phase, which reflects the fact that joint constraints of the two arms are mirror images (Figure 2A and B). When using their hands, participants preferred overhand grips, except at the extremes of the working range of motion, that is, orientations where they switched from grasping with the thumb on one side of the object to the other $\left(165^{\circ}\right.$ and $345^{\circ}$ for the left hand and $15^{\circ}$ and $195^{\circ}$ for the right hand; Figure $2 \mathrm{~B}$ ). Likewise, there was a $180^{\circ}$ phase shift in grip preference functions between the right and the left tool conditions (Figure 2B). As expected, given the differences in end-effector properties, grip preferences when using the tool differed significantly when compared with the hand on the same side. This was true for both the left side, $F(1,19)=$ $304.3, p<.001, M S E=175376.3$, and the right side, $F(1,19)=$ $239.7, p<.001, M S E=151407.6$. Not only did use of the tool introduce a $90^{\circ}$ offset between the opposition axis of the hand and that of the jaws, but it also caused the participants to change their preferred working range of motion compared with that of the hands. More precisely, while using almost exclusively pronated postures when grasping with their hands, participants often used supinated postures with the tool. This allowed them to avoid highly pronated postures and was likely due to the need to stabilize the tool against the forces of gravity by using the palm as a supportive platform for its weight.

Remarkably, the grips prospectively chosen in the PGS task reflected these constraints. As shown in Figure 2B, the functions representing grip preferences as a function of stimulus orientation for PGS and OGS tasks were virtually identical in each of the four conditions: Hand Left, $R=.90, F(1,10)=43.13, p<.0001$; Tool Left, $R=.99$, $F(1,10)=714.54, p<.0001 ;$ Hand Right, $R=.94, F(1,10)=$ 79.40, $p<.0001$; and Tool Right, $R=.99, F(1,10)=334.50$, $p<.0001$. This establishes that PGS judgments in both hand and tool conditions were based on internal representations that accurately capture the bio-mechanical properties of the limbs and the mechanical as well as the dynamical properties of the novel tool.

\section{fMRI Results}

\section{Planning Grasping Actions with the Hands or Tool Compared with Resting Baseline: Whole-Brain Analysis}

Relative to resting baseline, the pattern of activity increases associated with performance of the PGS task, in each of the four conditions defined by the 2 (Effector Type: hand, tool) $\times 2$ (Effector Side: left, right) design, was remarkably similar (Figure 3). As expected if the planning of grasping actions engages the parieto-frontal grasp circuit, aIPS (bilateral) and putative PMv (left) showed consistently increased activity regardless of whether grip preferences were based on the hand or the tool. This suggests that planning grasping actions with either the hands or the tool relied on the same regions. In all conditions, 


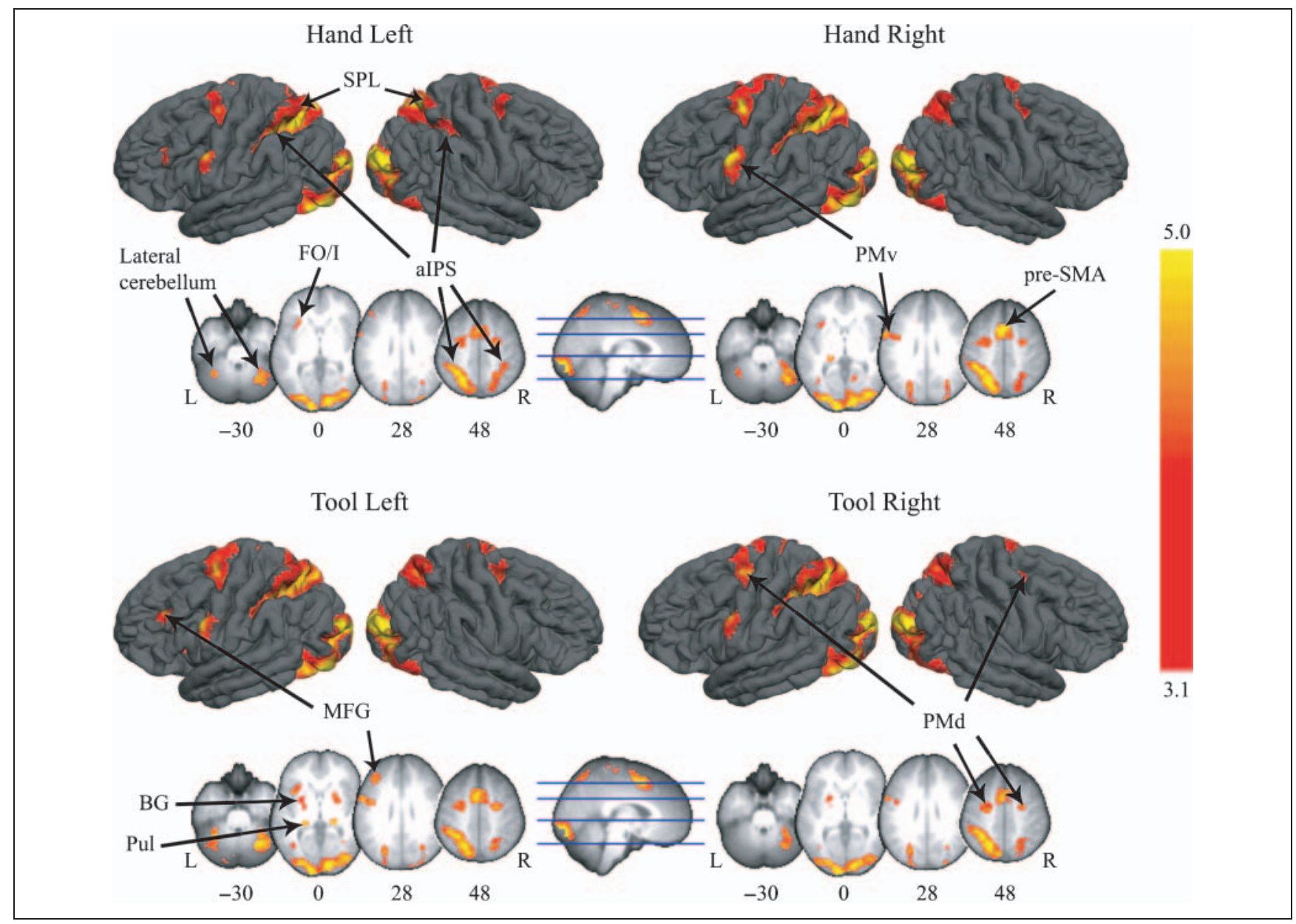

Figure 3. Increased neural activity associated with planning grasping actions with the hands or the tool relative to resting baseline revealed by whole-brain analyses. Group statistical parametric maps $(Z>3.1$, corrected cluster-extent significance threshold $p<.05)$ are overlaid on a 3-D surface reconstruction created from the 20 participants' anatomical scans (see the Methods section). Selecting grips (prospective grip selection [PGS] task) based on either hand or on the tool was consistently associated with activations in a strongly left lateralized neural network constituted of intraparietal sulcus (IPS), including its anterior sector (aIPS) and ventral premotor cortex (PMv) as well as in bilateral superior parietal lobule (SPL) and dorsal premotor cortex (PMd) and in pre-SMA. Right aIPS was activated only in the Hand Left condition. Other structures were also activated only in some conditions, such as left middle frontal gyrus (MFG), left frontal operculum/insula (FO/I), BG (globus pallidus and putamen), pulvinar nucleus (Pul), and lateral cerebellum. Increased activity in occipital regions was associated with use of visual stimuli. Data are also displayed on neurologically oriented axial (transverse) slices through ROIs. L = left hemisphere; $\mathrm{R}=$ right hemisphere. Slice coordinates are expressed relative to the vertical axis $(z)$ of the standard Montreal Neurological Institute space.

these effects were stronger in the left hemisphere irrespective of the effector side involved. We will return to these main findings shortly.

Increased activity was also detected in more dorsal regions, including the medial IPS and adjacent caudal superior parietal gyrus (putative parietal reach region; Connolly, Andersen, \& Goodale, 2003; Grafton, Fagg, Woods, $\&$ Arbib, 1996) and the intersection of the superior frontal and precentral sulci (putative dorsal premotor cortex [PMd]; Hoshi \& Tanji, 2000). These areas may constitute the human homologue of a circuit comprising the monkey superior parietal lobule and the PMd (or area F2), which transform objects' locations into motor programs for reaching and/or eye movements (Battaglia-Mayer et al., 2001; Marconi et al., 2001; Wise, Boussaoud, Johnson, \& Caminiti, 1997). These activations may thus reflect the internal simulation of reaching actions (Johnson et al., 2002). At least two alterna- tive interpretations exist for the role of PMd and caudal parietal cortex in the present task. One possibility is that these areas are directly involved in grip planning. In the macaque, PMd cells have been shown to code hand configuration during grasping (Hendrix, Mason, \& Ebner, 2009; Raos, Umiltá, Gallese, \& Fogassi, 2004). Likewise, recent findings indicate that cells in area V6A (located in the medial parieto-occipital cortex) are particularly involved in the coding of hand orientation during preparation and execution of handle grasping (Fattori et al., 2009). Another possibility is that these regions may be associated with the more abstract action selection demands of the PGS task (Schluter, Krams, Rushworth, \& Passingham, 2001; Hoshi, Shima, \& Tanji, 2000; Schluter, Rushworth, Passingham, \& Mills, 1998; Deiber et al., 1991).

Similar increases were found in the pre-SMA, which is involved in representing conditional visuomotor associations, 
for example, color and type of movement (Picard \& Strick, 2001). Bilateral increases in activity were also detected in the lateral cerebellum. This is consistent with earlier work implicating the cerebellar hemispheres in manual grasping (Grafton et al., 1996) and the acquisition of novel tool use skills (Imamizu et al., 2000). As discussed below, the cerebellum is also implicated in the predictive aspects of motor control, and the PGS task may involve the use of forward internal models. Finally, striate and extrastriate visual areas showed increases that are likely related to the processing of the stimulus objects.

A small number of regions showed significant increases in activity only when specific conditions were compared against baseline in the whole-brain analysis. These included motor-related subcortical structures within the BG (globus pallidus and putamen; bilateral in the Tool Left condition and left in the Tool Right condition) and the pulvinar nucleus of the thalamus (Tool Left condition, bilateral; Hand Right condition, left). Activity in the left middle frontal gyrus, which is involved in action selection (Schluter et al., 2001), was significantly increased only during Hand Left and Tool Left conditions. Finally, a cluster located at the border of the left frontal operculum and insula showed significant increases in all conditions except the Tool Right condition.

\section{Main Effects of Effector Type and Effector Side: Repeated Measures ANOVA}

Only those voxels that showed significantly increased activity relative to baseline in at least one of the four experimental conditions in the whole-brain analysis were considered further in a repeated measures ANOVA. Even when tested at a more liberal statistical threshold $(Z>$ 3.1, uncorrected for multiple comparisons), no significant main effects of either Effector Type or Effector Side were detected in parietal or frontal cortices. This failure cannot be attributed to insufficient statistical power, how- ever, as differences related to the main effect of side and to the interaction between both factors were unexpectedly detected in bilateral occipital cortex and left fusiform gyrus (Table 2). These are most likely attributable to uncontrolled Type I error.

\section{ROI Analyses}

To characterize the neural responses associated with grip planning within the grasp circuit further, we defined ROIs in putative left PMv and bilateral aIPS, as detailed in the Methods section. Mean percent signal change relative to the resting baseline (Figure 4) and mean time course of the BOLD signal (Figure S1 in supplemental material) were extracted from each ROI (see the Methods section).

Importantly, no differences were found between grip planning involving the hand versus the tool in any of the ROIs: main effect of Effector Type, $F(1,19)<1.0$ in all cases. By contrast, all ROIs were more active when grip selection decisions were based on the contralateral limb: main effect of Effector Side, left aIPS, $F(1,19)=4.3$, $p=.05, M S E=0.01$; right aIPS, $F(1,19)=14.5, p=.001$, $M S E=0.03$; left PMv, $F(1,19)=8.4, p=.009, M S E=$ 0.02 . In line with this observation, responses in the right aIPS (which was significantly activated with respect to resting baseline only in the Hand Left condition in the whole-brain analysis; Table 1 and Figure 3) differed from baseline only when the contralateral left limb was involved: Bonferroni-corrected one-tailed $t$ test, $t(19)=$ $3.96, p=.002$ and $t(19)=2.55, p=.04$ for the Hand Left and Tool Left conditions, respectively; $t(19)=1.98, p=$ .13 and $t(19)=1.21, p=.48$ for the Hand Right and Tool Right conditions, respectively. The main effect of Effector Side observed for all ROIs suggests that while the processes underlying grip planning within bilateral aIPS and putative left PMv did not differentiate between the

Table 2. Peak Coordinates of Clusters of Activations for 2 (Left, Right) $\times 2$ (Hand, Tool) Repeated measures ANOVA Analysis

\begin{tabular}{|c|c|c|c|c|}
\hline Contrast & & Cortical Region & $\begin{array}{c}\text { Peak Coordinates } \\
(x, y, z)\end{array}$ & Voxels \\
\hline $\begin{array}{l}\text { Main effect of Effector Type } \\
\text { (hand vs. tool) }\end{array}$ & - & - & - & - \\
\hline \multirow[t]{2}{*}{ Main effect of Effector Side } & Left $>$ Right & Right extrastriate occipital cortex & $16-9414$ & 87 \\
\hline & Right $>$ Left & Left extrastriate occipital cortex & $-8-986$ & 71 \\
\hline \multirow[t]{3}{*}{$\begin{array}{l}\text { Effector Type } \times \\
\quad \text { Effector Side interaction }\end{array}$} & $\begin{array}{l}\text { Crossed interaction: } \\
\text { Left: Tool > Hand }\end{array}$ & Left extrastriate occipital cortex & $-8-960$ & 65 \\
\hline & Right: Tool < Hand & & $-32-8410$ & 24 \\
\hline & & Left fusiform gyrus & $-30-54-20$ & 62 \\
\hline
\end{tabular}

Coordinates $(x, y, z)$ expressed in millimeters in the standard space of the Montreal Neurological Institute (MNI-152); $Z>3.1$, uncorrected for multiple comparisons, number of voxels $>10$. 
Figure 4. Percent signal change (PSC) relative to resting baseline associated with planning grasping actions in ROIs. The three ROIs, defined bilaterally in anterior intraparietal sulcus (aIPS) and in left ventral premotor cortex $(\mathrm{PMv})$, are displayed onto the average brain surface of the twenty participants (see the Methods section). For each of these regions, PSC averaged across subjects is shown for each condition. As whole-brain analyses showed a significant effect in right aIPS only in the Hand Left condition, the significance of PSC values for each condition was tested (one-tailed $t$ test using Bonferroni corrections) for this ROI. Error bars $=1$ SEM $* p<.05$. **Effector Side $\times$ Effector Type interaction $p<.05$.

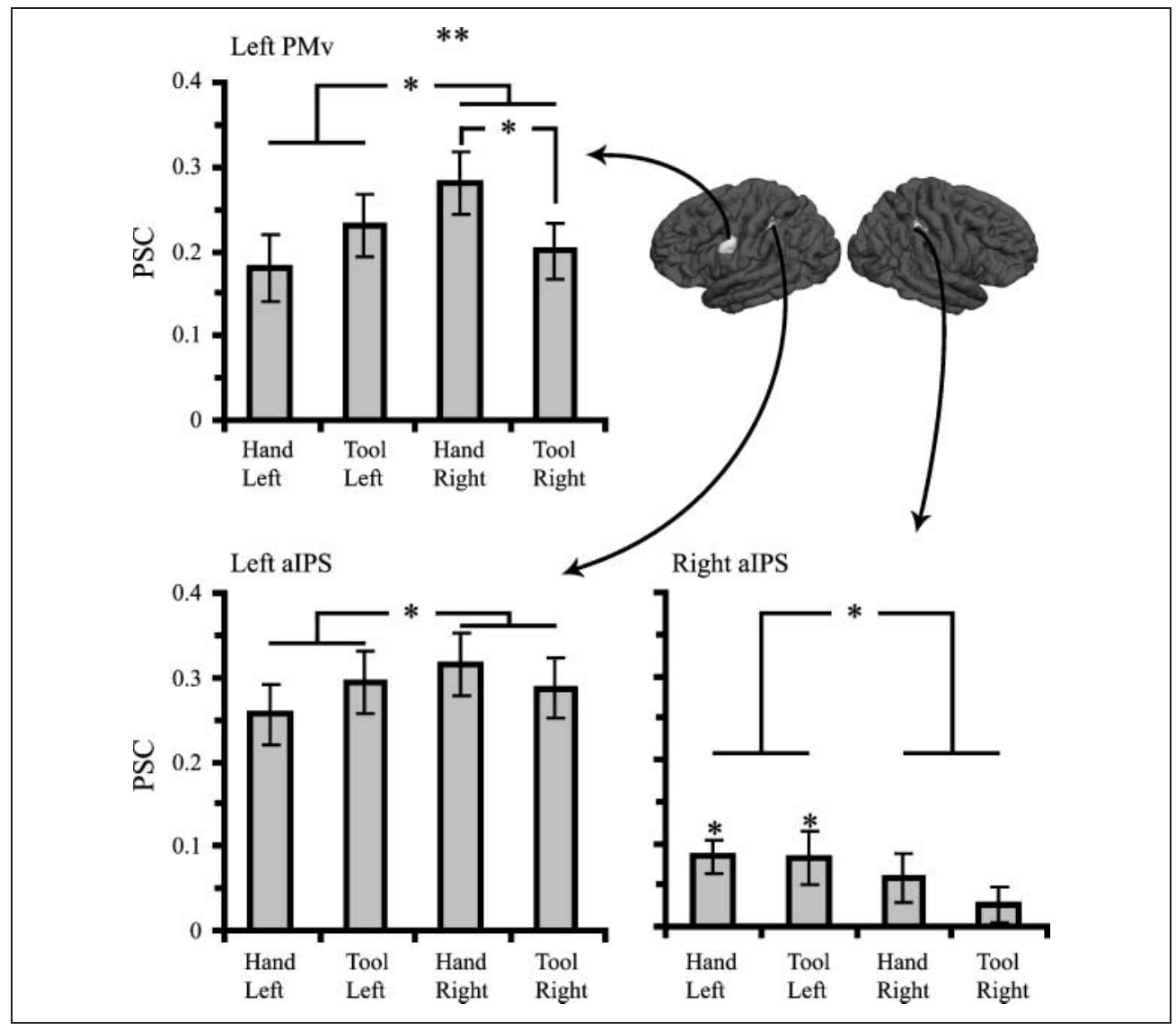

involvement of the hand or tool, they did evidence some degree of specificity for the contralateral limb. We return to this issue in the Discussion section.

Although all ROIs showed greater increases in activity for grip selection decisions on the basis of the contralateral side, responses in the putative grasp network showed a clear left cerebral asymmetry. As described above, left but not right PMv showed significant activation relative to baseline (Figure 3). Further analysis of potential subthreshold variations in activity within right PMv was performed post hoc on the basis of previous evidence of the engagement of this region during isometric grip (Ehrsson et al., 2000, 2001) and grip-lift tasks (Davare et al., 2006) performed with the right hand (see Figure S2 in supplemental material for detailed information). In all conditions, activity within this region did not differ from resting baseline: Hand Left, $t(1,19)=-0.55, p=.58$; Tool Left, $t(1,19)=$ $0.07, p=.94$; Hand Right, $t(1,19)=1.20, p=.24$; Tool Right, $t(1,19)=0.09, p=.93$. As for aIPS, it was consistently activated above baseline in the left hemisphere, whereas the increase in activity reached significance on the right side only when the contralateral left limb was involved (Figures 3 and 4). Direct comparison between activity in left and right aIPS confirmed this apparent left cerebral asymmetry regardless of whether grip selection decisions were based on the left or right side, two-tailed $t$ test, $t(79)=14.52, p<.0001$.
Finally, the interaction between Effector Type and Effector Side was nonsignificant in aIPS of both hemispheres: left aIPS, $F(1,19)=1.7, p=.2$; right aIPS, $F(1$, $19)<1.0$. By contrast, this interaction was significant in putative left PMv, $F(1,19)=9.0, p=.007$, MSE $=0.08$ (Figure 4$)$, reflecting the fact that responses were greater for the hand than the tool when the right limb was involved, Bonferroni-corrected two-tailed $t$ test, $t(19)=-2.9, p=$ .018 , whereas they did not differ between effector types when the left side was involved, $t(19)=1.4, p=.35$.

\section{DISCUSSION}

This study yielded two major results. First, the behavioral findings indicate that the initial practice session was sufficient to establish a new internal representation that captured the unique end-effector properties of the novel tool. Second, despite behavioral evidence for the use of effector-specific representations, planning grasping actions based on the hands or the tool nevertheless engaged the very same regions of parietal and frontal cortex, including areas (aIPS and putative PMv) of the previously described grasp circuit. Together, these behavioral and neuroimaging findings support the hypothesis that grasping objects with a tool is supported by the same areas that represent manual prehension. Each of these points is considered in detail below along with their implications. 


\section{Planning Grasping Actions with the Hands or Tool Relies on Effector-specific Representations}

After practice, participants expressed grip preferences based on the use of either the hands or the tool that accurately corresponded to those demonstrated earlier during actual prehension (Figure 2B). Critically, these preferences reflected the unique mechanical properties of the tool, namely, the $90^{\circ}$ offset between its opposition axis and that of the hand. In addition, prospective grip preferences reflected the dynamical constraints associated with use of the tool, as illustrated by the change in preferred range of motion between grasps executed with the tool versus the hand in both PGS and OGS tasks (Figure 2B). These observations indicate that grip selection decisions in the PGS task relied on internal representations that accurately captured the specific physical properties of the newly mastered device as well as the respective bio-mechanical constraints of the limbs. Further, the fact that participants were able to switch between the hand and the tool successfully when selecting grips (Figure 1D) indicates that the effector-specific representations on which these decisions were based coexist in a relatively independent manner. As discussed below, these facts support the hypothesis that PGS performances are based on the same internal representations involved in the selection of overt grasping actions performed either with the hand or tool. Variations in BOLD signal recorded during performance of the PGS task should therefore provide insights into these mechanisms.

\section{Planning Grasping with the Hands or Tool Involves Common Parieto-frontal Mechanisms}

Functional MRI results suggest that planning grasping actions based on the tool or hands increased activity within the very same regions of parietal and premotor cortex, most notably putative homologues of the macaque grasp circuit (aIPS and PMv). In other words, following the development of expertise using the tool, the very same brain regions appear to represent grasping actions involving either the tool or the hands. Further, both PMv and aIPS showed stronger responses within the motor dominant left hemisphere. This cerebral asymmetry occurred irrespective of the limb involved in the task and is consistent with the well-known dominance of the left hemisphere for a variety of praxis behaviors (Kroliczak \& Frey, 2009; Johnson-Frey, Newman-Norlund, \& Grafton, 2005; Rothi \& Heilman, 1997; Kimura \& Archibald, 1974).

Three sources of evidence support the claim that grasping actions involving the hands or the tool are represented in the same parieto-frontal areas. First, relative to the resting baseline, grip selection based on either the hands or the tool was associated with a strikingly similar pattern of increased neural activity. In addition to the hypothesized involvement of the grasp circuit (i.e., aIPS and PMv), activations were also detected in posterior parietal cortex (within and along the IPS), in caudal superior parietal lobule, and in PMd (Figure 3). Second, when directly tested for a main effect of effector type, none of the parietal or frontal regions that were activated during grip selection showed any significant differences between hand and tool. Finally, although more sensitive ROI analyses did detect various degrees of specificity for the contralateral versus ipsilateral limb within aIPS (bilateral) and PMv (left), they did not reveal a main effect of the type of effector used. In fact, the only evidence of a potential differential coding of grasps planned with the tool or hand comes from the interaction between effector type and side within left PMv. This interaction reflected higher activity when planning grasps with the right hand versus with the tool held in the right hand. This result seems to suggest that, in right-handers, this ventral frontal region may support distinct representations for grasping with the hand versus tool when the dominant limb is involved. An alternative interpretation would be that higher activity when planning grasps with the hand might reflect a stronger involvement in coding precision grips, performed when grasping the object with the hand, than in controlling power grasps, as used to manipulate the tool. This grip-related effect has previously been demonstrated in manual grasping (Ehrsson et al., 2000). However, both interpretations imply either a main effect of the type of effector in putative left PMv or a similar involvement of right putative PMv for grasps planned on the basis of the left arm. The fact that neither was observed in the present data makes both interpretations unlikely, and further work will be needed to determine the precise role of left PMv in planning grasps with the hand and a tool.

Previous neuroimaging studies investigating the neural bases of precision grip formation reported a bilateral engagement of $\mathrm{PMv}$ for grasps performed with the right hand (Ehrsson et al., 2000, 2001), whereas transcranial magnetic stimulation of left and right PMv disrupts positioning of the fingers on the object during a precision grip-lift task (Davare et al., 2006). One likely explanation for the lack of right PMv recruitment in any condition in the present study is that the present task only involved planning grasping actions rather than overt execution. The left hemisphere might play a dominant role in those processes, regardless of the limb involved (Kuhtz-Buschbeck et al., 2003). The absence of differences between the patterns of brain activity associated to planning grasps with the hand (using a precision grip) and with the tool (whose manipulation required the use of a power grasp) might appear somewhat surprising based on previous evidence showing differential neural recruitment for overt precision and power grasps (Ehrsson et al., 2000). However, recent work suggests that these findings might in fact reflect differences in the force engaged in both types of overt grasps (Kuhtz-Buschbeck et al., 2008). It is therefore likely that such differences would not arise at the level of planning grasping actions, as investigated in the present task. 


\section{Forward Internal Models of Grasping}

One potential criticism of this work is that, during fMRI, we relied exclusively on a planning task (PGS) that did not involve subsequent movement execution. That is, planning under these circumstances might involve internal representations that differ from those engaged when planning overt grasping. It is certainly true that the descending efferent commands and presence of afferent feedback during overt grasping would engage additional neural mechanisms (Gerardin et al., 2000). However, at least two sources of evidence suggest that prospective planning tasks are a valid way to assess premovement representations. First, there is considerable evidence that the selection of the macroscopic features of grasping (e.g., under vs. overhand) is actually completed before movement onset (Stelmach, Castiello, \& Jeannerod, 1994; Arbib, 1981; Jeannerod, 1981; Keele, 1981). The internal representations underlying such selection should therefore be available even in tasks that do not demand overt execution. Second, the compatibility between grip preferences expressed in the PGS and OGS tasks was established empirically through psychophysical testing. As detailed earlier, we found a striking similarity between PGS and OGS functions relating grip preferences for the hands and tool to stimulus orientations (Figure 2B). Remarkably, grip preferences in PGS even reflected the different working range of motion evident in OGS, as participants sought to avoid less stable grips when manipulating the tool. This approach was furthermore based on previous work suggesting that both overt and prospective action-planning tasks rely on common internal representations (Johnson, 1998, 2000) and neural substrates (Buxbaum, Johnson-Frey, \& Bartlett-Williams, 2005; Johnson et al., 2002; Johnson, Corballis, \& Gazzaniga, 2001). The nearly identical pattern of grip preferences expressed in OGS and PGS tasks is consistent with the idea that grip selection relies on a forward internal model that accurately predicts the sensory consequences of grasping movements involving the hands or tool (Wolpert \& Flanagan, 2001). The model for this previously unfamiliar tool was presumably acquired during the overt practice phase. Internal simulation of these models would provide an opportunity to select the least bio-mechanically awkward grip option on the basis of predicted sensory feedback in advance or in the absence of overt movement execution (Johnson, 2000). Both cerebellar and posterior parietal mechanisms have been implicated in forward internal models (Shadmehr \& Krakauer, 2008; Wolpert \& Flanagan, 2001; Imamizu et al., 2000), and the involvement of these regions in the present task may be viewed as reflecting these predictive processes.

\section{Implications for Motor Equivalence and Tool Use Abilities}

As noted at the outset, previous neuroimaging studies identified overlapping patterns of brain activity when the same action was performed with different biological effectors (Castiello et al., 2000; Rijntjes et al., 1999). These findings were interpreted as evidence for effectorindependent levels of representation that are responsible for regularities in motor behaviors undertaken with different effectors (i.e., motor equivalence; Wing, 2000; Keele et al., 1990). In the present whole-brain analysis, planning grasping actions based on the hand or tool also led to indistinguishable patterns of increased neural activity in a variety of regions, including aIPS and PMv. However, a critical distinction with respect to the motor equivalence neuroimaging work is that the psychophysical dissociations in our behavioral data clearly indicate that planning was based on effector-specific representations of the hand or tool. Therefore, our findings suggest that planning grasping actions either with the hand or with the tool are underpinned by effector-specific representations that are implemented within the same parieto-frontal areas. Together, these results establish that common patterns of neural activation across different effectors are a necessary, but not sufficient, source of evidence for the existence of effectorindependent levels of action representation. The interpretation instead depends entirely on the accompanying behavioral evidence. Furthermore, it has to be noted again that our results concern only the planning stage of grasping actions; they therefore do not exclude the possibility that partially distinct patterns of activations might accompany the execution of grasps with the tool as compared with the hand.

The apparent incongruity between our behavioral and neuroimaging results can be understood in several different ways. A first possibility is that effector-specific representations for either the hand or the tool are actually coded in discrete subregions within these parieto-frontal areas, but that the resolution of conventional fMRI is simply insufficient to detect these differences. The interaction observed in left PMv between effector type and side, with a more sensitive ROI approach, might reflect such a segregation. However, the fact that this difference between hand and tool occurs only for the right hand, when the behavioral evidence for effector specificity applies equally to both sides, is inconsistent with this view. Alternatively, there is ample evidence from single-unit electrophysiology for both effector-specific and effectorindependent motor representations present within the same regions of parietal (Fogassi et al., 2005; Hyvarinen, 1982) and PMv (Rizzolatti et al., 1988) cortices. On the basis of this precedent, increased activity in these same regions when planning actions in accordance with the unique properties of the hand or tool is therefore not unexpected.

As mentioned in the Introduction section, recent evidence shows that after training, neurons in the monkey PMv and primary motor cortex (F1) that code manual grasps come to also represent grasping actions performed with a tool. Importantly, this is true regardless of the particular hand movements involved in manipulation 
of the tool (Umiltà et al., 2008). This suggests that, as a result of extensive training, individual neurons develop representations of grasp that are independent of whether the monkey uses the hand or tool. Although we do not have direct evidence for this, it is tenable that similar changes accompanied training in the present experiment and that the activations we observed also reflected increased activity in such effector-independent representations. Indeed, a recent fMRI study showed similar activations in aIPS when grasping with the hand or a tool, and these data could also be interpreted as evidence for effector independence (Gallivan, Cavina-Pratesi, \& Culham, 2009). However, our psychophysical findings demonstrate that, at least in the human, training results in the acquisition of effector-specific representations of grasping with the tool that are functionally independent of existing representations of manual grasping. Further, our data show that effector specificity emerges upstream from primary motor cortex within the parieto-frontal network subserving prehension and temporally at the stage of premovement planning.

To conclude, the present results demonstrate that grasping actions involving the hands or a newly mastered tool depend on relatively independent internal representations that are supported by the very same brain areas. Considered in the context of recent single-unit data, these results suggest that the development of tool use expertise is accompanied by two types of experiencedependent changes within the putative PMv and aIPS grasp circuit. The first, illustrated by the current findings, is the establishment of effector-specific representations of grasping with the tool that coexist with effector-specific representations of manual grasps. This level of representation is critical for accurate planning and execution of actions in a manner that is sensitive to the unique properties of these effectors. The second, reflected by the recent findings of Umiltà et al. (2008), is the acquisition of effector-independent representations through the modification of existing representations of the hand. This more abstract level of representation may facilitate the transfer of skills between hand and tool and may have implications for understanding adaptation to upper extremity prostheses.

\section{Acknowledgments}

The authors would like to thank Prof. M. Arbib for the fruitful discussion of a previous version of the manuscript. This work has been supported by grants to S. H. F. from the National Institute of Neurological Disorders and Stroke (No. NS053962) and from the Army Research Laboratory (No. 49581-LS). S. J. was supported by the Fyssen Foundation, and C. D. was supported by the Alexander von Humboldt Foundation.

Reprint requests should be sent to Stéphane Jacobs, Espace et Action-INSERM U864, 16 rue du Doyen Lépine, 69676 Bron cedex, France, or via e-mail: stephane.jacobs@inserm.fr or Scott H. Frey, Department of Psychology, 1227 University of Oregon, Eugene, OR 97403, or via e-mail:shfrey@uoregon.edu.

\section{REFERENCES}

Arbib, M. A. (1981). Perceptual structures and distributed motor control. In V. B. Brooks (Ed.), Handbook of physiology-The nervous system (Vol. II, pp. 1449-1480). Oxford: Oxford University Press.

Battaglia-Mayer, A., Ferraina, S., Genovesio, A., Marconi, B., Squatrito, S., Molinari, M., et al. (2001). Eye-hand coordination during reaching: II. An analysis of the relationships between visuomanual signals in parietal cortex and parieto-frontal association projections. Cerebral Cortex, 11, 528-544.

Beckmann, C. F., Jenkinson, M., \& Smith, S. M. (2003). General multilevel linear modeling for group analysis in fMRI. Neuroimage, 20, 1052-1063.

Binkofski, F., Buccino, G., Stephan, K. M., Rizzolatti, G., Seitz, R. J., \& Freund, H. J. (1999). A parieto-premotor network for object manipulation: Evidence from neuroimaging. Experimental Brain Research, 128, 210-213.

Buxbaum, L. J., Johnson-Frey, S. H., \& Bartlett-Williams, M. (2005). Deficient internal models for planning hand-object interactions in apraxia. Neuropsychologia, 43, 917-929.

Castiello, U., \& Begliomini, C. (2008). The cortical control of visually guided grasping. Neuroscientist, 14, 157-170.

Castiello, U., Bennett, K. M., Egan, G. F., Tochon-Danguy, H. J., Kritikos, A., \& Dunai, J. (2000). Human inferior parietal cortex "programs" the action class of grasping. Cognitive Systems Research, 1, 89-97.

Connolly, J. D., Andersen, R. A., \& Goodale, M. A. (2003). fMRI evidence for a "parietal reach region" in the human brain. Experimental Brain Research, 153, 140-145.

Culham, J. C., Danckert, S. L., DeSouza, J. F., Gati, J. S., Menon, R. S., \& Goodale, M. A. (2003). Visually guided grasping produces fMRI activation in dorsal but not ventral stream brain areas. Experimental Brain Research, 153, 180-189.

Dale, A. M., Fischl, B., \& Sereno, M. I. (1999). Cortical surfacebased analysis: I. Segmentation and surface reconstruction. Neuroimage, 9, 179-194.

Davare, M., Andres, M., Clerget, E., Thonnard, J. L., \& Olivier, E. (2007). Temporal dissociation between hand shaping and grip force scaling in the anterior intraparietal area. Journal of Neuroscience, 27, 3974-3980.

Davare, M., Andres, M., Cosnard, G., Thonnard, J. L., \& Olivier, E. (2006). Dissociating the role of ventral and dorsal premotor cortex in precision grasping. Journal of Neuroscience, 26, 2260-2268.

Deiber, M. P., Passingham, R. E., Colebatch, J. G., Friston, K. J., Nixon, P. D., \& Frackowiak, R. S. (1991). Cortical areas and the selection of movement: A study with positron emission tomography. Experimental Brain Research, 84, 393-402.

Duvernoy, H. M. (1991). The buman brain: Surface, blood supply, and three-dimensional sectional anatomy (2nd ed.). New York: Springer Wien.

Ehrsson, H. H., Fagergren, A., Jonsson, T., Westling, G., Johansson, R. S., \& Forssberg, H. (2000). Cortical activity in precision- versus power-grip tasks: An fMRI study. Journal of Neurophysiology, 83, 528-536.

Ehrsson, H. H., Fagergren, E., \& Forssberg, H. (2001). Differential fronto-parietal activation depending on force used in a precision grip task: An fMRI study. Journal of Neurophysiology, 85, 2613-2623.

Fattori, P., Breveglieri, R., Marzocchi, N., Filippini, D., Bosco, A., \& Galletti, C. (2009). Hand orientation during reach-to-grasp movements modulates neuronal activity in the medial posterior parietal area V6A. Journal of Neuroscience, 29, 1928-1936.

Fischl, B., Sereno, M. I., \& Dale, A. M. (1999). Cortical surfacebased analysis: II. Inflation, flattening, and a surface-based coordinate system. Neuroimage, 9, 195-207. 
Fischl, B., Sereno, M. I., Tootell, R. B. H., \& Dale, A. M. (1999). High-resolution inter-subject averaging and a coordinate system for the cortical surface. Human Brain Mapping, 8, 272-284.

Fogassi, L., Ferrari, P. F., Gesierich, B., Rozzi, S., Chersi, F., \& Rizzolatti, G. (2005). Parietal lobe: From action organization to intention understanding. Science, 308, 662-667.

Fogassi, L., Gallese, V., Buccino, G., Craighero, L., Fadiga, L., \& Rizzolatti, G. (2001). Cortical mechanism for the visual guidance of hand grasping movements in the monkey-A reversible inactivation study. Brain, 124, 571-586.

Frey, S. H., Vinton, D., Norlund, R., \& Grafton, S. T. (2005). Cortical topography of human anterior intraparietal cortex active during visually guided grasping. Brain Research, Cognitive Brain Research, 23, 397-405.

Gallivan, J. P., Cavina-Pratesi, C., \& Culham, J. (2009). Is that within reach? fMRI reveals that the human superior parietooccipital cortex encodes objects reachable by the hand. Journal of Neuroscience, 29, 4381-4391.

Gentilucci, M., Roy, A. C., \& Stefanini, S. (2004). Grasping an object naturally or with a tool: Are these tasks guided by a common motor representation? Experimental Brain Research, 157, 496-506.

Gerardin, E., Sirigu, A., Lehericy, S., Poline, J. B., Gaymard, B., Marsault, C., et al. (2000). Partially overlapping neural networks for real and imagined hand movements. Cerebral Cortex, 10, 1093-1104.

Grafton, S. T., Fagg, A. H., Woods, R. P., \& Arbib, M. A. (1996). Functional anatomy of pointing and grasping in humans. Cerebral Cortex, 6, 226-237.

Hendrix, C., Mason, C., \& Ebner, T. (2009). Signaling of grasp dimension and grasp force in dorsal premotor cortex and primary motor cortex neurons during reach to grasp in the monkey. Journal of Neurophysiology, 102, 132-145.

Hoshi, E., Shima, K., \& Tanji, J. (2000). Neuronal activity in the primate prefrontal cortex in the process of motor selection based on two behavioral rules. Journal of Neurophysiology, 83, 2355-2373.

Hoshi, E., \& Tanji, J. (2000). Integration of target and body-part information in the premotor cortex when planning action. Nature, 408, 466-470.

Hyvarinen, J. (1982). Posterior parietal lobe of the primate brain. Physiological Reviews, 62, 1060-1129.

Imamizu, H., Miyauchi, S., Tamada, T., Sasaki, Y., Takino, R., Putz, B., et al. (2000). Human cerebellar activity reflecting an acquired internal model of a new tool. Nature, 403, 192-195.

Jeannerod, M. (1981). The neural and behavioral organization of goal-directed movements (Vol. 10). New York: Oxford Science Publishers.

Jeannerod, M., Arbib, M. A., Rizzolatti, G., \& Sakata, H. (1995). Grasping objects. The cortical mechanisms of visuomotor transformation. Trends in Neurosciences, 18, 205-320.

Jenkinson, M., Bannister, P., Brady, M., \& Smith, S. (2002). Improved optimization for the robust and accurate linear registration and motion correction of brain images. Neuroimage, 17, 825-841.

Jenkinson, M., \& Smith, S. (2001). A global optimisation method for robust affine registration of brain images. Medical Image Analysis, 5, 143-156.

Johnson, S. H. (1998). Cerebral organization of motor imagery: Contralateral control of grip selection in mentally represented prehension. Psychological Science, 9, 219-222.

Johnson, S. H. (2000). Thinking ahead: The case for motor imagery in prospective judgements of prehension. Cognition, 74, 33-70.

Johnson, S. H., Corballis, P. M., \& Gazzaniga, M. S. (2001). Within grasp but out of reach: Evidence for a double dissociation between imagined hand and arm movements in the left cerebral hemisphere. Neuropsychologia, 39, 36-50.

Johnson, S. H., Rotte, M., Grafton, S. T., Hinrichs, H., Gazzaniga, M. S., \& Heinze, H. J. (2002). Selective activation of a parietofrontal circuit during implicitly imagined prehension. Neuroimage, 17, 1693-1704.

Johnson-Frey, S. H., Newman-Norlund, R., \& Grafton, S. T. (2005). A distributed left hemisphere network active during planning of everyday tool use skills. Cerebral Cortex, 15, 681-695.

Keele, S. W. (1981). Motor control. In W. B. Brooks (Ed.), Handbook in physiology: Section 1. The nervous system (Vol. 2, pp. 1391-1414). Baltimore: Williams \& Williams.

Keele, S. W., Cohen, A., \& Ivry, R. (1990). Motor programs: Concepts and issues. In M. Jeannerod (Ed.), Attention and performance 13: Motor representation and control (pp. 77-110). Hillsdale, NJ: Lawrence Erlbaum Associates.

Kimura, D., \& Archibald, Y. (1974). Motor functions of the left hemisphere. Brain, 97, 337-350.

Kroliczak, G., \& Frey, S. H. (2009). A common network in the left cerebral hemisphere represents planning of tool use pantomimes and familiar intransitive gestures at the handindependent level. Cerebral Cortex, 19, 2396-2410.

Kuhtz-Buschbeck, J. P., Gilster, R., Wolff, S., Ulmer, S., Siebner, H., \& Jansen, O. (2008). Brain activity is similar during precision and power gripping with light force: An fMRI study. Neuroimage, 40, 1469-1481.

Kuhtz-Buschbeck, J. P., Mahnkopf, C., Holzknecht, C., Siebner, H., Ulmer, S., \& Jansen, O. (2003). Effector-independent representations of simple and complex imagined finger movements: A combined fMRI and TMS study. European Journal of Neuroscience, 18, 3375-3387.

Lashley, K. S. (1930). Basic neural mechanisms in behavior. Psychological Review, 37, 1-24.

Logothetis, N. K., Pauls, J., Augath, M., Trinath, T., \& Oeltermann, A. (2001). Neurophysiological investigation of the basis of the fMRI signal. Nature, 412, 150-157.

Marconi, B., Genovesio, A., Battaglia-Mayer, A., Ferraina, S., Squatrito, S., Molinari, M., et al. (2001). Eye-hand coordination during reaching. I. Anatomical relationships between parietal and frontal cortex. Cerebral Cortex, 11, 513-527.

Miezin, F. M., Maccotta, L., Ollinger, J. M., Petersen, S. E., \& Buckner, R. L. (2000). Characterizing the hemodynamic response: Effects of presentation rate, sampling procedure, and the possibility of ordering brain activity based on relative timing. Neuroimage, 11, 735-759.

Mumford, J. (2007). A guide to calculating percent change with Featquery. Unpublished technical report. Retrieved from http://mumford.bol.ucla.edu/perchange_guide.pdf.

Oldfield, R. C. (1971). The assessment and analysis of handedness: The Edinburgh Inventory. Neuropsychologia, 9, 97-113

Picard, N., \& Strick, P. L. (2001). Imaging the premotor areas. Current Opinion in Neurobiology, 11, 663-672.

Raos, V., Umiltá, M., Gallese, V., \& Fogassi, L. (2004). Functional properties of grasping-related neurons in the dorsal premotor area F2 of the macaque monkey. Journal of Neurophysiology, 92, 1990-2002.

Rice, N. J., Tunik, E., \& Grafton, S. T. (2006). The anterior intraparietal sulcus mediates grasp execution, independent of requirement to update: New insights from transcranial magnetic stimulation. Journal of Neuroscience, 26, 8176-8182.

Rijntjes, M., Dettmers, C., Buchel, C., Kiebel, S., Frackowiak, R. S., \& Weiller, C. (1999). A blueprint for movement: Functional and anatomical representations in the human motor system. Journal of Neuroscience, 19, 8043-8048. 
Rizzolatti, G., Camarda, R., Fogassi, L., Gentilucci, M., Luppino, G., \& Matelli, M. (1988). Functional organization of inferior area 6 in the macaque monkey: II. Area F5 and the control of distal movements. Experimental Brain Research, 71, 491-507.

Rothi, L. J. G., \& Heilman, K. M. (1997). Apraxia. The neuropsychology of action. Hove, UK: Psychology Press.

Schluter, N. D., Krams, M., Rushworth, M. F. S., \& Passingham, R. E. (2001). Cerebral dominance for action in the human brain: The selection of actions. Neuropsychologia, 39, 105-113.

Schluter, N. D., Rushworth, M. F., Passingham, R. E., \& Mills, K. R. (1998). Temporary interference in human lateral premotor cortex suggests dominance for the selection of movements. A study using transcranial magnetic stimulation. Brain, 121, 785-799.

Segonne, F., Dale, A. M., Busa, E., Glessner, M., Salvolini, U., Hahn, H. K., et al. (2004). A hybrid approach to the skullstripping problem in MRI. Neuroimage, 22, 1060-1075.

Shadmehr, R., \& Krakauer, J. W. (2008). A computational neuroanatomy for motor control. Experimental Brain Research, 185, 359-381.

Smith, S. M. (2002). Fast robust automated brain extraction. Human Brain Mapping, 17, 143-155.

Stelmach, G. E., Castiello, U., \& Jeannerod, M. (1994). Orienting the finger opposition space during prehension movements. Journal of Motor Behavior, 26, 178-186.
Tunik, E., Frey, S. H., \& Grafton, S. T. (2005). Virtual lesions of the anterior intraparietal area disrupt goal-dependent on-line adjustments of grasp. Nature Neuroscience, 8, 505-511.

Umiltà, M. A., Escola, L., Intskirveli, I., Grammont, F., Rochat, M., Caruana, F., et al. (2008). When pliers become fingers in the monkey motor system. Proceedings of the National Academy of Sciences, U.S.A., 105, 2209-2213.

Wing, A. M. (2000). Motor control: Mechanisms of motor equivalence in handwriting. Current Biology, 10, R245-R248.

Wise, S. P., Boussaoud, D., Johnson, P. B., \& Caminiti, R. (1997). Premotor and parietal cortex: Corticocortical connectivity and combinatorial computations. Annual Review of Neuroscience, 20, 25-42.

Wolpert, D. M., \& Flanagan, J. R. (2001). Motor prediction. Current Biology, 11, R729-R732.

Woolrich, M. W., Behrens, T. E. J., Beckmann, C. F., Jenkinson, M., \& Smith, S. M. (2004). Multi-level linear modelling for fMRI group analysis using Bayesian inference. Neuroimage, 21, 1732-1747.

Woolrich, M. W., Ripley, B. D., Brady, J. M., \& Smith, S. M. (2001). Temporal autocorrelation in univariate linear modelling of fMRI data. Neuroimage, 14, 1370-1386.

Worsley, K. J., Evans, A. C., Marrett, S., \& Neelin, P. (1992). A three-dimensional statistical analysis for CBF activation studies in human brain. Journal of Cerebral Blood Flow and Metabolism, 12, 900-918. 